\title{
HOPE: HOme Power Efficiency System for a Green Network
}

\author{
Han Yan $^{\ddagger *}$, Fabrice Fontaine ${ }^{\ddagger}$, Olivier Bouchet ${ }^{\ddagger}$, Jean-Paul Vuichard ${ }^{\ddagger}$, Jean-Philippe Javaudin ${ }^{\ddagger}$, Maryline Lebouc ${ }^{\ddagger}$, \\ Marie-Helene Hamon ${ }^{\ddagger}$, Cedric Gueguen*, Bernard Cousin* \\ ${ }^{\ddagger}$ France Télécom \{first name.surname\} @orange.com \\ *University of Rennes1/IRISA \{first name.surname\}@irisa.fr
}

\begin{abstract}
A home network represents an increasingly large portion of a household's electrical consumption due to the increasing number of digital home devices and the increasing complexity of the underlying communication infrastructure. Limiting a home network's power consumption is a priority for large institutions as witnessed by evolving regulation, and a priority for individual consumers wishing to reduce their electric bills. In this demonstration, we proposed a solution that aims at reducing power consumption at both the device and network level. At the device level, our system can turn the devices off when they are not in operation and quickly turn them on when they are needed. At the network level, the solution is based on a new convergence layer denoted inter-MAC, which provides a common infrastructure to the different home network paths. In particular, the inter-MAC can be used to choose a greener path. Our solution on both levels is compatible and complementary to home network energy saving.
\end{abstract}

Keywords-Efficient energy saving; Green networking; ZigBee; UPnP Low Power; UPnP Audio Video; Heterogeneous network, Convergence layer; Green path selection; Digital home.

\section{INTRODUCTION}

In the last decade, there has been a proliferation of connected devices in the home environment. The number of connected devices has led to a sharp increase in energy consumption. A home network [1] is a complex environment which can contain several different types of devices: Set-Top Box (STB), Home GateWay (HGW), network attached storage (NAS), laptop, power line communication (PLC) plugs and so on; which are interconnected with different kinds of connections: Wi-Fi, Ethernet and power line communication. These home network devices represent an increasingly large portion of a homes electric consumption, which in turn generates greater carbon emissions and the costs consumers more.

For these two reasons, environmental and economical, it is essential to provide an effective means of reducing power consumption, which is also transparent for the user. To do so, we propose a technical solution on two levels. The first device level solution uses an overlay control network which switches existing devices on or off according to their usage. The second network level solution allows for selecting the least amount of energy to be consumed or least radiating a data path among the available links (Wi-Fi, Ethernet, PLC etc.).

These two phases are compatible and could be combined into a single offering. The standardization process is underway, with initial contributions proposed by standards bodies (HGI [2], IEEE P1905 [3]).

\section{System ARChitecture}

Our HOPE system proposes two solutions for energy saving. The solution at the device level could change the power levels of each device depending on its usage in a simple network. Since our home network connections are becoming increasingly complex, one device could accommodate several connections. This is why the second solution works at the network level: it selects the greener path.

\section{A. The device-level system}

The device-level system for reducing power consumption is based on an energy control layer overlaying the home network. This energy control layer can turn off the devices or switch the devices to a very low power consumption mode when they are not in operation. Moreover, the system can automatically wake up the devices when the user needs them. During this process, all energy control messages are sent on a low-power consumption network like ZigBee [4] or by using a low-power consumption protocol like UPnP Low Power [5].

All the different home network devices are considered HOPE nodes in our system. As shown in Fig. 1, a HOPE system is composed of two kinds of functional blocks: service blocks and low power consumption connection blocks. Service functional blocks intercept the service request required by the actual device, and then send this request through the low power consumption control layer.

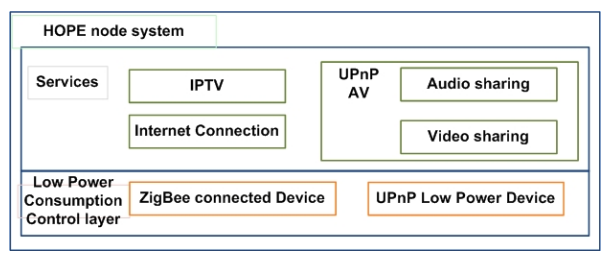

Fig. 1. Device-level HOPE node architecture

In our demonstration, the system is able to satisfy basic services such as an IPTV or Internet connection. Furthermore, there are several enriched multimedia services, such as audio/video sharing, which are implemented by UPnP AV [6], which demonstrates the efficiency of our energy saving system for collaborative services. Since these sharing services are collaborative services, the actual device requires more than a network connection service on the HGW: it also requires 
turning on a second device and activating the audio/video sharing services on the second device.

\section{B. The network-level system}

The proposed network-level system for reducing power consumption is based on a technology-independent layer, called an inter-MAC, from the Omega project [7], [8].

This inter-MAC convergence layer is positioned above the different MAC layer of the different technologies. The interMAC layer is composed of three functional planes (Fig. 2): the control plane, the data plane and the management plane. The data plane is responsible for frame forwarding. The control plane is responsible for short-term decisions related to the establishment and release of paths and the processing of upper layer requests. It is divided into several engines: QoS, Monitoring, Inter-MAC Adapter and Green Path Selection.

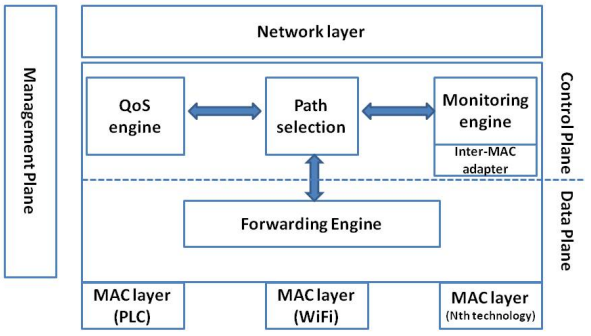

Fig. 2. Network-level HOPE inter-MAC architecture

The green path selection protocol takes into account the following three metrics: Available bandwidth, energy consumption, and radio frequency. More technical details are presented in [9].

\section{DESCRIPTION OF DEMONSTRATIONS}

In the first part of our demonstration shown in Fig. 3, the HOPE system aims to maintain the devices in a low-power consumption state when the devices are not in use. Meanwhile, if the devices are required by a service, it can be woken up by our HOPE system. For example, in the IPTV scenario, a user wants to watch IPTV on his STB. The STB is connected to the HGW through a pair of PLC plugs. When the user turns the STB on to watch IPTV, the requirement of establishing a network connection through the PLC plugs is intercepted by our system. The PLC plugs are woken up and a network connection for the IPTV service is established. When the user turns the STB off, the PLC plugs are also turned off since the connection from the HGW to the STB is not needed.

Several scenarios are presented in this section to demonstrate the efficiency of our system on enriched services.

Fig. 4 represents a heterogeneous home network configuration with Ethernet, PLC and WiFi links. The second part of our demonstration contains 4 mini PCs, which play the role of extenders; they implement the green path selection protocol. The end devices (including a PC, NAS, TV, tablet and mobile phone) are connected to these extenders. The Home Gateway connects the home network to the Internet.

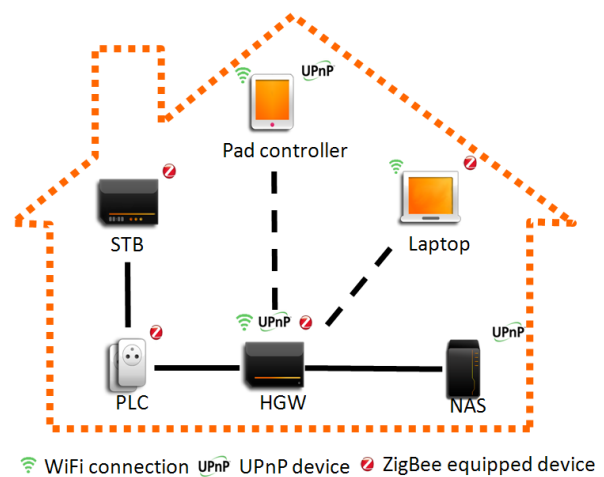

Fig. 3. Device-level HOPE demonstration

The idea is to highlight energy consumption reduction while maintaining satisfactory QoS for different flows, due to the green path selection protocol introduced in section II.

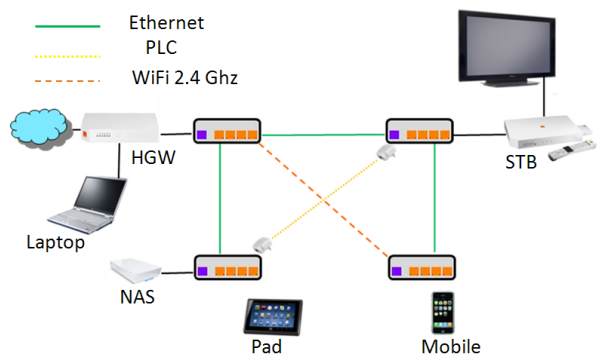

Fig. 4. Network level HOPE demonstration

\section{CONCLUSION}

Our solution at two levels, described in this demonstration, can drastically reduce the consumption of home networks. Users can benefit from enriched home multimedia services and efficiently manage their power consumption. Our future work will study the reduction of power consumption combining both the device component level and the network level in different services in order to further reduce the total power consumption of the home network.

\section{ACKNOWLEDGMENT}

This work was supported by the French government in the framework of the FUI10 Econhome project.

\section{REFERENCES}

[1] B. Rose, "Home networks: a standards perspective," Communications Magazine, IEEE, vol. 39, no. 12, pp. 78 -85, 2001.

[2] $H G I$, http://www.homegatewayinitiative.org/ Std.

[3] IEEE P1905.1, http://grouper.ieee.org/groups/1905/1/ Std.

[4] Zigbee Specifications, Z. Alliance Std., 2008.

[5] UPnP Low Power Architecture, UPnP Forum Std., 2008.

[6] UPnP Audio Video Architecture, UPnP Forum Std., 2002.

[7] ICT OMEGA, http://www.ict-omega.eu Std.

[8] V. Suraci, A. Cimmino, R. Colella, G. Oddi, and M. Castrucci, "Convergence in home gigabit networks: Implementation of the inter-mac layer as a pluggable kernel module," in PIMRC, IEEE, 2010.

[9] O. Bouchet, A. Kortebi, and M. Boucher, "Inter-mac green path selection for heterogeneous networks," in GLOBECOM, workshop HeterWMN, IEEE, 2012. 